\title{
SUM OF TWO FOURTH POWERS OF INTEGERS
}

\author{
MINORU TSUNEKAWA
}

Introduction. Problems concerning the sum of two fourth powers of integers seem to be so difficult that little has been known since long years [3]. For instance, it is an important problem to determine whether there are infinitely many prime numbers which are represented in the form $p=a^{4}+b^{4}$. But nothing is known except that the density of such prime numbers is easily proved to be 0 ; accordingly it is difficult to obtain a necessary and sufficient condition under which $p$ is represented in such a form.

In this paper we propose to derive several theorems on the above subject by investigating the sum of two fourth powers of integers in the biquadratic number field $R(\sqrt{i})$ or in its subfields. We shall use as main tools the decomposition law of prime numbers in $R(\sqrt{i})$ and the concrete expression of a fundamental unit in $R(\sqrt{i})$.

Hereinafter we say that an integer in a certain number field is of $B$ type if it is represented as a sum of two fourth powers of integers belonging to the field and we denote by B. P. "the representation as a sum of two fourth powers of integers".

The contents of $\S 1$ relate to the existence problem of $B$ type prime numbers in the subfield of $R(\sqrt{i})$ different from the rational number field, $\S 2$ to the uniqueness problem of B. P. and $\S 3$ to B. P. of the product of several $B$ type prime numbers.

Here the writer would like to express his thanks to Prof. S. Kuroda and Dr. T. Kubota for their instructive advices.

\section{Notations and Preliminaries}

(1) $i=\sqrt{-1}, R=$ rational number field.

(2) Minimal basis of $R(i), R(\sqrt{2}), R(\sqrt{-2})$ or $R(\sqrt{i})$ are respectively (1, $i),(1, \sqrt{2}),(1, \sqrt{-2})$ or $(1, \sqrt{i}, i, i \sqrt{i})$, and their class numbers are all 1 .

Received November 22, 1960. 
Hence every ideal in each field is principal.

(3) The fundamental unit in $R(\sqrt{ } i)$, as well as $R(\sqrt{ } 2)$, is $\varepsilon=1+\sqrt{ } 2$. For the sake of convenience, we put $\sqrt{i}=(1+i) / \sqrt{ } 2$, and so $\varepsilon=1+\sqrt{2}=1+$ $(1-i) \sqrt{i}$. Hence, for $n=0, \pm 1, \pm 2, \ldots, \varepsilon^{n}=r+s(1-i) \sqrt{i}$, where $r$ and $s$ are rational integers satisfying $r^{2}-2 s^{2}=(-1)^{n}$.

(4) Necessary and sufficient condition under which a rational prime number $p$ is completely decomposed in $R(\sqrt{i})$ is $p \equiv 1(\bmod 8)$.

(5) The notation $a \mid b$ or $a+b$ respectively means that $a$ is a divisor of $b$ or not, where $a$ and $b$ are integers.

$\S 1$. Concerning the existence of $B$ type prime numbers, we have the following results.

Theorem 1. There is no $B$ type prime number $(\notin R)$ in $R(i)$.

Proof. Let a prime number $\pi(\notin R)$ in $R(i)$ be of $B$ type and put

$$
\pi=\alpha^{4}+\beta^{4}=(\alpha+\beta \sqrt{i})(\alpha-\beta \sqrt{i})(\alpha+\beta i \sqrt{i})(\alpha-\beta i \sqrt{i}) ; \alpha, \beta \in R(i)
$$

Since then $\pi$ is prime in $R(i)$, at least two factors in the right hand side of (1.1) must be of the form $\pm(\sqrt{i})^{k} \varepsilon^{n}$, where $k=0,1,2$ or 3 and $n=0, \pm 1$, $\pm 2, \ldots$ Suppose, for instance, $\alpha+\beta \sqrt{i}= \pm(\sqrt{i})^{k} \varepsilon^{n}$ and put $\varepsilon^{n}=r+s(1-i) \sqrt{i}$, then

$$
\alpha+\beta \sqrt{i}= \pm(\sqrt{i})^{k}\{\gamma+s(1-i) \sqrt{i}\}
$$

Therefore

(1) If $k=0$, then $\alpha= \pm r, \beta= \pm s(1-i)$

(2) If $k=1$, then $\alpha= \pm s(1+i), \beta= \pm r$

(3) If $k=2$, then $\alpha= \pm r i, \beta= \pm s(1+i)$

(4) If $k=3$, then $\alpha=\mp s(1-i), \beta= \pm r i$.

In these four cases, we have $\pi= \pm\left(r^{4}-4 s^{4}\right)$ after all. But this is contrary to the assumption $\pi \notin R$. Next we suppose that the left hand side of (1.2) is equal to $\alpha-\beta \sqrt{i}, \alpha+\beta i \sqrt{i}$ or $\alpha-\beta i \sqrt{i}$. In these cases proof will be similarly carried out by interchanging $\beta$ with $-\beta$ or with $\pm \alpha$.

THEOREM $1^{\prime}$. The only $B$ type prime numbers $(\notin R)$ in $R(\sqrt{2})$ are $5 \varepsilon^{4 m}$ $(m= \pm 1, \pm 2, \ldots)$ and there is no $B$ type prime number $(\notin R)$ in $R(\sqrt{-2})$. 
Proof. In the case of $R(\sqrt{ } 2)$, let a prime number $\pi(\notin R)$ in $R(\sqrt{2})$ be of $B$ type and put

$$
\pi=\alpha^{4}+\beta^{4}=(\alpha+\beta \sqrt{ } i)(\alpha-\beta \sqrt{ } i)(\alpha+\beta i \sqrt{ } i)(\alpha-\beta i \sqrt{ } i),
$$

where $\alpha, \beta \in R(\sqrt{2})$ and $(\alpha, \beta)=1$. Since then $\pi$ is prime in $R(\sqrt{2})$, at least two factors in the right hand side of (1.3) must be of the form $\pm(\sqrt{ } i)^{k} \varepsilon^{m}$, where $k=0,1,2$ or 3 and $m=0, \pm 1$, $2, \ldots$ First we treat the case:

$$
\alpha+\beta \sqrt{i}= \pm(\sqrt{i})^{k} \varepsilon^{m}
$$

It is easily seen that (1.4) is impossible for $k=0,1$, so we closely examine the remaining cases of $k=2,3$.

(1) If $k=2$, then taking square of the both sides of (1.4) we have $2 \alpha \beta \sqrt{i}$ $=-\left(\varepsilon^{2 m}+\alpha^{2}+\beta^{2} i\right)$, whence

$$
4 \alpha^{2} \beta^{2} i=\left(\varepsilon^{2 m}+\alpha^{2}\right)^{2}-\beta^{4}+2 \beta^{2}\left(\varepsilon^{2 m}+\alpha^{2}\right) i .
$$

Since $\alpha, \beta$ and $\varepsilon \in R(\sqrt{2})$ and $\beta \neq 0$, we obtain $\alpha^{2}=\varepsilon^{2 m}$ and so $\beta^{2}=2 \varepsilon^{2 m}$. Hence $\pi=\alpha^{4}+\beta^{4}=5 \varepsilon^{4 m}(m \neq 0)$.

(2) If $k=3$, then (1.4) gives the relation $\alpha= \pm(\sqrt{i})^{3} \varepsilon^{m}-\beta \sqrt{i}$. Hence $\alpha^{2}=\left(\beta^{2}-\varepsilon^{2 m}\right) i \pm 2 \varepsilon^{m} \beta$. This means $\alpha^{2}= \pm 2 \varepsilon^{m} \beta$ and $\beta^{2}-\varepsilon^{2 m}=0$. Consequently $\beta^{4}=\varepsilon^{4 m}, \alpha^{4}=4 \varepsilon^{4 m}$ and $\pi=\alpha^{4}+\beta^{4}=5 \varepsilon^{4 m}(m \neq 0)$.

Assuming that the left hand side of (1.4) is respectively equal to $\alpha-\beta \sqrt{ } i$, $\alpha+\beta i \sqrt{i}$ or $\alpha-\beta i \sqrt{i}$, the proof will be similarly carried out through interchanging $\beta$ with $-\beta$ or $\pm \alpha$.

Accordingly it has been decided that $\pi=5 \varepsilon^{4 m}$ is a necessary condition for a prime number $\pi$ in $R(\sqrt{2})$ to be of $B$ type. This is clearly sufficient, for $5 \varepsilon^{4 m}=\left(2 \varepsilon^{m}\right)^{4}+\left(\varepsilon^{m}\right)^{4}$.

In the case of $K(\sqrt{-2})$, we have (1.4) with $\alpha, \beta \in R(\sqrt{-2})$ and can conclude quite similarly that $\pi=5 \varepsilon^{4 m}(m \neq 0)$ are the only $B$ type prime numbers in $R(\sqrt{-2})$. But $5 \varepsilon^{4 m} \notin R(\sqrt{-2})$.

THEOREM $1^{\prime \prime}$. There is no $B$ type prime number $(\notin R, R(\sqrt{2}))$ in $R(\sqrt{ } i)$.

The proof of this theorem shall well be omitted, because it is essentially the same as in the case of theorem 1 in spite of a comparatively complicated computation.

§2. It seems very difficult to determine in a general form the number of 
B. P. of a given integer in a field. Until now the following results with regard to a product of two prime numbers has only been obtained.

THEOREM 2. Let $r, s$ be rational prime numbers which are either 2 or of the form $8 h+1$ and further let the product rs be of $B$ type, then the $B . P$. of rs is unique.

Proof. Assume $r s=x_{1}^{4}+y_{1}^{4}=x_{2}^{4}+y_{2}^{4}$ under the condition $\left(x_{1}, y_{1}\right)=\left(x_{2}, y_{2}\right)$ $=1$ and consider the following decompositions in $R(\sqrt{i})$,

$$
\begin{aligned}
& x_{1}^{4}+y_{1}^{4}=\left(x_{1}+y_{1} \sqrt{i}\right)\left(x_{1}-y_{1} \sqrt{i}\right)\left(x_{1}+y_{1} i \sqrt{i}\right)\left(x_{1}-y_{1} i \sqrt{i}\right) . \\
& x_{2}^{4}+y_{2}^{4}=\left(x_{2}+y_{2} \sqrt{i}\right)\left(x_{2}-y_{2} \sqrt{i}\right)\left(x_{2}+y_{2} i \sqrt{i}\right)\left(x_{2}-y_{2} i \sqrt{i}\right) .
\end{aligned}
$$

On the other hand, decompose respectively $r, s$ into $r=\pi \bar{\pi}, s=\sigma \bar{\sigma}$ in $R(i)$, and respectively $\pi, \bar{\pi}, \sigma$, and $\bar{\sigma}$ into $\pi=\pi_{1} \pi_{2}, \bar{\pi}=\bar{\pi}_{1} \bar{\pi}_{2}, \sigma=\sigma_{1} \sigma_{2}$ and $\bar{\sigma}=\bar{\sigma}_{1} \bar{\sigma}_{2}$ in $R(\sqrt{i})$ : i.e.

$$
\begin{aligned}
& r=\pi \bar{\pi}=\pi_{1} \pi_{2} \bar{\pi}_{1} \bar{\pi}_{2} \\
& s=\sigma \bar{\sigma}=\sigma_{1} \sigma_{2} \bar{\sigma}_{1} \bar{\sigma}_{2} .
\end{aligned}
$$

Now each factor in the right hand sides of (2.3) and (2.4) is distributed into each factor of the right hand sides of (2.1) and (2.2). (Consider the norm from $R(\sqrt{i})$ to $R$ ). Notations being suitably selected, we may assume that

$$
\pi_{1} \sigma_{1} !\left(x_{1}+y_{1} \sqrt{i}\right) .
$$

Here let us examine other factors ;

(1) Suppose first that $\pi_{1} \sigma_{1}$ is also contained in a factor of (2.2), for instance, in $x_{2}+y_{2} \sqrt{i}$. Then, using (2.5), we obtain $\pi_{1} \sigma_{1} \mid\left(x_{1} y_{2}-x_{2} y_{1}\right)$ which causes the following relation

$$
r s \mid\left(x_{1} y_{2}-x_{2} y_{1}\right)
$$

As $\left|x_{1} y_{2}-x_{2} y_{1}\right|<r s$ unless $\left|x_{1}\right|=\left|y_{1}\right|=\left|x_{2}\right|=\left|y_{2}\right|=1$, it follows from (2.6) that $x_{1} y_{2}-x_{2} y_{1}=0$, which yields $x_{2}= \pm x_{1}, y_{2}= \pm y_{1}$, for $\left(x_{1}, y_{1}\right)=\left(x_{2}, y_{2}\right)=1$. Accordingly B. P. is unique. If $\pi_{1} \sigma_{1}$ is contained in one of any other three factors of (2.2) than $x_{2}+y_{2} \sqrt{i}$, a similar proof is available.

(2) Suppose secondly that $\pi_{1} \sigma_{1}$ is not contained in any factor of (2.2). Then, notations being suitably chosen, we can assume

$$
\pi_{1} \sigma_{2} \mid\left(x_{2}+y_{2} \sqrt{i}\right) \text {. }
$$


Therefore, using (2.5) and (2.7), we have

$$
r \mid\left(x_{1} y_{2}-x_{2} y_{1}\right)
$$

similarly to the above case (1). Here again we closely examine the factor of (2.2) which contains $\sigma_{1}$.

(i) If $\sigma_{1} \mid\left(x_{2}-y_{2} \sqrt{i}\right)$, then from (2.5) we obtain $s \mid\left(x_{1} y_{2}+x_{2} y_{1}\right)$ and the relation $r s \mid\left\{\left(x_{1} y_{2}\right)^{2}-\left(x_{2} y_{1}\right)^{2}\right\}$ through (2.8). Since $\left|\left(x_{1} y_{2}\right)^{2}-\left(x_{2} y_{1}\right)^{2}\right|<\left(x_{1} y_{2}\right)^{2}$ $+\left(x_{2} y_{1}\right)^{2}<\frac{1}{2}\left(x_{1}^{4}+y_{2}^{4}+x_{2}^{4}+y_{1}^{4}\right)=r s$, we have $\left(x_{1} y_{2}\right)^{2}=\left(x_{2} y_{1}\right)^{2}$. Therefore $x_{2}$ $= \pm x_{1}, y_{2}= \pm y_{1}$.

(ii) If $\sigma_{1} \mid\left(x_{2}+y_{2} i \sqrt{ } i\right)$, then $s \mid\left(x_{1} x_{2}+y_{1} y_{2}\right)$ after all. Hence, $r s \mid\left(x_{1} x_{2}+y_{1} y_{2}\right)$ $\left(x_{1} y_{2}-x_{2} y_{1}\right)$ holds by (2.8). Since $\left|\left(x_{1} x_{2}+y_{1} y_{2}\right)\left(x_{1} y_{2}-x_{2} y_{1}\right)\right| \leqq \frac{1}{2}\left\{\left(x_{1} x_{2}+y_{1} y_{2}\right)^{2}\right.$ $\left.+\left(x_{1} y_{2}-x_{2} y_{1}\right)^{2}\right\}<r s$, we have $\left(x_{1} x_{2}+y_{1} y_{2}\right)\left(x_{1} y_{2}-x_{2} y_{1}\right)=0$, which leads to the same conclusion.

(iii) If $\sigma_{1} \mid\left(x_{2}-y_{2} i \sqrt{ } i\right)$, then a similar proof is available.

(3) In other remaining cases where $\pi_{1} \sigma_{2}$ is contained in any one of ( $x_{2}$ $\left.-y_{2} \sqrt{ } i\right),\left(x_{2}+y_{2} i \sqrt{ } i\right)$ or $\left(x_{2}-y_{2} i \sqrt{ } i\right)$, we can also obtain similar proofs.

Theorem 2'. If a product $\pi \sigma(\notin R)$ of two prime numbers $\pi, \sigma$ in $R(i)$ is of $B$ type, then its $B$. P. is unique.

Proof. Under conditions $\alpha, \beta \in R(i)$ and $(\alpha, \beta)=1$, we put

$$
\pi \sigma=\alpha^{4}+\beta^{4}=\left(\alpha^{2}+\beta^{2} i\right)\left(\alpha^{2}-\beta^{2} i\right) .
$$

If $(1+i, \pi \sigma)=1$, then $\left(\alpha^{2}+\beta^{2} i, \alpha^{2}-\beta^{2} i\right)=1$ and if $(1+i, \pi \sigma) \neq 1$, then $\alpha^{2}+\beta^{2} i$ and $\alpha^{2}-\beta^{2} i$ have the common factor $1+i$ at least, and so $\pi \sigma= \pm(1+i)^{2}$ $= \pm 2 i$. If further any one of the factors in (2.9) is \pm 1 or $\pm i$, then, quite similarly to (1.2), we get $\pi \sigma= \pm\left(r^{4}-4 s^{4}\right)$. This is, however, contrary to the assumption $\pi \sigma \notin R$. Hence $\pi$ and $\sigma$ must be contained separately in two factors of (2.9). If $\pi= \pm\left(\alpha^{2}+\beta^{2} i\right)$ and $\sigma= \pm\left(\alpha^{2}-\beta^{2} i\right)$, then

$$
\alpha^{2}= \pm(\pi+\sigma) / 2, \quad \beta^{2}=\mp i(\pi-\sigma) / 2 .
$$

If $\pi= \pm i\left(\alpha^{2}+\beta^{2} i\right), \sigma=\mp i\left(\alpha^{2}-\beta^{2} i\right)$, then

$$
\alpha^{2}= \pm i(\pi-\sigma) / 2, \quad \beta^{2}= \pm(\pi+\sigma) / 2 .
$$

The latter can be obtained from (2.10) by interchanging $\alpha$ with $\beta$. Thus, $\alpha$ and $\beta$ are uniquely determined through (2.10). 
Note. There are many examples which show that the above theorems 2, $2^{\prime}$ do not necessarily hold for a product of more than two prime numbers.

Ex. $1 . \quad 17 \cdot 63113 \cdot 80537=542^{4}+103^{4}=514^{4}+359^{4}$

Ex. 2. $2 \cdot 113 \cdot 4889 \cdot 2953=239^{4}+7^{4}=227^{4}+157^{4}$

Ex. 3. $(1+4 i)(7-8 i)(3+20 i)=(10+3 i)^{4}+(9-5 i)^{4}$

$$
=(5+2 i)^{4}+3^{4}(1+i)^{4} \text {. }
$$

§. It is also a hard problem to determine generally whether a product of several given $B$ type prime numbers has B. P. or not. First let us state a preliminary lemma without proof.

Lemma. Let a product $N=p_{1} p_{2} \cdots p_{n}$ of different $B$ type rational prime numbers $p_{m}=a_{m}^{4}+b_{m}^{4}(m=1,2, \ldots, n)$ be of $B$ type and put

$$
N=a^{4}+b^{4}, \quad(a, b)=1,
$$

then the following relation holds;

$$
\prod_{m=1}^{n}\left(a_{m}+b_{m} \sqrt{ } i\right)= \pm(\sqrt{ } i)^{k} \varepsilon^{l}(a+b \sqrt{i}),
$$

where $k=0,1,2$ or $3, l=0, \pm 1, \pm 2, \ldots$ and $\varepsilon^{l}=r+s(1-i) \sqrt{ } i$. Without any loss of generality, the following conditions can be added:

$$
a>0, \quad b>0, \quad 2 \mid b, \quad a_{m}>0, \quad r>0 .
$$

Under these conditions the right hand side of (3.2) is written as follows:

$$
\prod_{m=1}^{n}\left(a_{m}+b_{m} \sqrt{i}\right)= \pm(\sqrt{ } i)^{k}\{r a+s b+(r b+s a) \sqrt{ } i+s b i-s a i \sqrt{ } i\}
$$

For $N=2 p_{1} p_{2} \cdots p_{n}$, we have similarly

$$
\text { (1+i) } \begin{aligned}
& \prod_{m=1}^{n}\left(a_{m}+b_{m} \sqrt{ } i\right) \\
& = \pm(\sqrt{ } i)^{k}\{r a+s b+(r b+s a) \sqrt{ } i+s b i-s a i \sqrt{ } i\}
\end{aligned}
$$

where $a b$ is to be odd.

TheOREM 3. Notations being as in the preceding lemma, none of the products $p_{1} p_{2}, p_{1} p_{2} p_{3}, 2 p_{1}, 2 p_{1} p_{2}$ and $2 p_{1} p_{2} p_{3}$ can be of $B$ type.

Proof. In the case of $N=p_{1} p_{2},(3.4)$ gives 


$$
\begin{aligned}
& \left(a_{1}+b_{1} \sqrt{i}\right)\left(a_{2}+b_{2} \sqrt{i}\right) \\
& \quad= \pm(\sqrt{i})^{k}\{r a+s b+(r b+s a) \sqrt{i}+s b i-s a i \sqrt{ } i\} .
\end{aligned}
$$

Now let us closely examine four cases of $k=0,1,2$ and 3 .

(1) If $k=0$, then, since $(1, \sqrt{i}, i, i \sqrt{i})$ is a basis of $R(\sqrt{i})$, the following relations hold $(\rho= \pm 1)$ :

$$
\begin{aligned}
\rho(r a+s b) & =a_{1} a_{2} \\
\rho(r b+s a) & =a_{1} b_{2}+a_{2} b_{1} \\
\rho s b & =b_{1} b_{2} \\
-\rho s a & =0 .
\end{aligned}
$$

From the last formula of (3.7) we have $s=0$, which is contrary to the assumption $b_{1} \neq 0, b_{2} \neq 0$.

(2) If $k=1$, then we have $\rho s b=0$ and $\rho s a=a_{1} a_{2}$, which is a contradiction.

(3) If $k=2$, then the following relations hold:

$$
\begin{aligned}
\rho(r a+s b) & =b_{1} b_{2} \\
\rho(r b+s a) & =0 \\
-\rho s b & =a_{1} a_{2} \\
\rho s a & =a_{1} b_{2}+a_{2} b_{1} .
\end{aligned}
$$

Here $s=0$ is impossible, for $a_{1} a_{2} \neq 0$. If $|s| \geqq 2$, then it follows from the 3 rd and 4 th formulas of (3.8) that

$$
b \leqq \frac{1}{2} a_{1} a_{2}, \quad a \leqq \max \left(\left|a_{1} b_{2}\right|,\left|a_{2} b_{1}\right|\right) .
$$

But these can not be true, for $a^{4}+b^{4}=\left(a_{1} a_{2}\right)^{4}+\left(a_{1} b_{2}\right)^{4}+\left(a_{2} b_{1}\right)^{4}+\left(b_{1} b_{2}\right)^{4}$. Accordingly $|s|=1$, but this is also impossible from the 2nd relation of (3.8).

(4) If $k=3$, the proof is similar to the case (3).

In the case of $N=p_{1} p_{2} p_{3}$, (3.4) yields

(3.10) $\prod_{m=1}^{3}\left(a_{m}+b_{m} \sqrt{ } i\right)= \pm(\sqrt{i})^{k}\{r a+s b+(r b+s a) \sqrt{ } i+s b i-s a i \sqrt{ } i\}$.

Put, for convenience,

$$
\begin{aligned}
& A=a_{1} a_{2} a_{3} \\
& B=b_{1} a_{2} a_{3}+b_{2} a_{3} a_{1}+b_{3} a_{1} a_{2} \\
& C=a_{1} b_{2} b_{3}+a_{2} b_{3} b_{1}+a_{3} b_{1} b_{2} \\
& D=b_{1} b_{2} b_{3} .
\end{aligned}
$$


Then one and only one of $A, B, C$ and $D$ is odd, because $a_{1} b_{1}, a_{2} b_{2}, a_{3} b_{3}$ are all even. Now let us examine four cases of $k=0,1,2$ and 3 .

(1) If $k=0$, then it follows from (3.10) and (3.11), that

$$
\begin{aligned}
\rho(r a+s b) & =A \\
\rho(r b+s a) & =B \\
\rho s b & =C \\
-\rho s a & =D .
\end{aligned}
$$

If $s$ is odd in (3.12), then $A$ and $D$ are odd, since $b$ is even. This is, however, contrary to the fact mentioned above. Hence $s$ must be even. If $s=0$, then $D=b_{1} b_{2} b_{3}=0$, which can not hold. If $|s| \geqq 4$, then the 3rd and 4 th formulas of (3.12) give

$$
a \leqq \frac{1}{4}\left|b_{1} b_{2} b_{3}\right|, \quad b \leqq \frac{3}{4} \max \left(\left|a_{1} b_{2} b_{3}\right|, \quad\left|a_{2} b_{3} b_{1}\right|, \quad\left|a_{3} b_{1} b_{2}\right|\right),
$$

which can not hold by a quite similar reason as (3.9) did not. Finally suppose $|s|=2$, then the 1st formula of (3.12) implies $2\left|a_{1} a_{2} a_{3}, 8\right| D, 8 \mid s a$ and $4 \mid a$, which contradicts the assumption $2+a$.

(2) If $k=1$, we have $\rho s b=D$ and $\rho s a=A$, which is impossible.

(3) If $k=2$, then (3.10) gives

$$
\left\{\begin{aligned}
\rho(r a+s b) & =C \\
\rho(r b+s a) & =D \\
-\rho s b & =A \\
\rho s a & =B .
\end{aligned}\right.
$$

Here $s$ must be even from a similar reason to the case of $k=0$. But neither $s=0$ nor $|s| \geqq 4$ can hold. Hence $|s|=2(r=3)$. Now by eliminating $a, b, a_{3}$ and $b_{3}$ from (3.13), we obtain the relation

$$
\left(r^{2}-s^{2}\right) a_{1}^{2} a_{2}^{2}-r s\left(a_{1} b_{2}+a_{2} b_{1}\right)\left(a_{1} a_{2}+b_{1} b_{2}\right)+s^{2}\left\{b_{1}^{2} b_{2}^{2}+\left(a_{1} b_{2}+a_{2} b_{1}\right)^{2}\right\}=0 .
$$

Put $s=2 \rho_{1}\left(\rho_{1}= \pm 1\right)$ and $r=3$, and further put $\left(a_{1} / b_{1}\right)=t_{1}$ and $\left(a_{2} / b_{2}\right)=t$. Then by an easy computation the above relation turns out

$$
\left(5 t_{2}^{2}+6 \rho_{1} t_{2}+4\right) t_{1}^{2}+2 \rho_{1}\left(3 t_{2}^{2}+4 \rho_{1} t_{2}+3\right) t_{1}+4 t_{2}^{2}+6 \rho_{1} t_{2}+4=0 .
$$

Now we can easily prove that (3.14) can not hold for any real values of $t_{1}$ and $t_{2}$. For, first of all, $5 t_{2}^{2}+6 \rho_{1} t_{2}+4>0$, and, $D$ being the discriminant with 
respect to $t_{1}$ of the left hand side of (3.14), we have

$$
D=\left(3 t_{2}^{2}+4 \rho_{1} t_{2}+3\right)^{2}-\left(5 t_{2}^{2}+6 \rho_{1} t_{2}+4\right)\left(4 t_{2}^{2}+6 \rho_{1} t_{2}+4\right),
$$

where $5 t_{2}^{2}+6 \rho_{1} t_{2}+4>3 t_{2}^{2}+4 \rho_{1} t_{2}+3$ and $4 t_{2}^{2}+6 \rho_{1} t_{2}+4>3 t_{2}^{2}+4 \rho_{1} t_{2}+3$. Hence $D<0$.

(4) If $k=3$, then a similar relation to (3.13) leads to a similar conclusion.

By means of (3.5) we can accomplish an almost same proof in the case of $N=2 p$, or $2 p_{1} p_{2}$ and a comparatively complicated but analogous one in the case of $N=2 p_{1} p_{2} p_{3}$.

Here we want to add a supplementary corollary and theorem derived almost immediately from theorem 3 and theorem $1^{\prime}$.

Corollary. $p_{1}^{2}, p_{1}^{3}$ and $p_{1}^{2} p_{2}$ cannot be of $B$ type.

Proof. Clear, because the lemma is valid for $n \leqq 3$ even if $p_{m}$ are not necessarily different.

Note. This corollary can, however, not be extended in general, for example, if $p_{1}=a_{1}^{4}+b_{1}^{4}$, then $p_{1} p_{2}^{4}=\left(a_{1} p_{2}\right)^{4}+\left(b_{1} p_{2}\right)^{4}$.

Theorem 3'. A product $\nu=\pi_{1} \pi_{2} \cdots \pi_{n}$ of $B$ type prime numbers $\pi_{m}(m$ $=1,2, \ldots, n)$ in $R(\sqrt{2})$ has B. P. in $R(\sqrt{2})$, if and only if $n=4 h+1$.

This theorem is well comprehended without proof, because it is easily seen that $\nu$ must be of the form $5^{n} \varepsilon^{4 k}$ from theorem $1^{\prime}$ and factors $2+i$ and $2-i$ of 5 are prime in $R(\sqrt{i})$.

Note. We can imagine that theorem 3 may not be extended in general, but for the product of four $B$ type rational primes the theorem seems also to be true.

\section{REFERENCES}

[1] S. Kuroda, Über den Dirichletschen Körper, Jour. of the Faculty of Science, University of Tokyo, Sec 1, vol. IV, Part 5 (1943), pp. 384-393.

[2] T. Takagi, Daisûteki Seisûron (The theory of algebraic numbers), Tokyo, 1948.

[3] L. E. Dickson, History of the theory of numbers, vol. 2(1920), pp. 644-648.

\section{Nagoya Institute of Technology}

